\title{
ON THE FORMS OF QUINTIC SCROLLS.
}

\author{
BY DR. VIRGIL SNYDKR.
}

(Read before the American Mathematical Society, February 22, 1902.)

The only paper devoted to the systematic study of quintic scrolls is that by Schwarz, * in which surfaces are classified according to the configuration of the double curve. Fifteen types are enumerated, of which ten are unicursal, four of genus 1, and one of genus 2. Schwarz regarded the scroll as generated by the line of intersection of corresponding planes in two developables the sum of whose classes is 5 .

It seems desirable to enumerate the types from the dual standpoint, $i$. e., regarded as generated by the line joining corresponding points of two plane or twisted curves. Of course, the same forms will appear that were obtained before, but the relations between subforms are more clearly brought out.

\section{A. Unicursal Quintics.}

Let a line $\delta$ and a unicursal quartic $c_{4}$ be put in $(1,1)$ correspondence and lines joining corresponding points be drawn; these lines will generate a quintic scroll having $\delta$ for a simple director line; any plane through $\delta$ will cut the scroll in 4 generators which intersect in 6 points and each cuts $\delta$ in one point. The plane is a fourfold tangent plane ; hence the double curve is of order 6 (type II). $\dagger$ The double curve does not cut $\delta$.

Let $\delta$ cut $c_{4}$, then a generator coincides with $\delta$; the residual curve is of order 5 , it cuts $\delta$ in 3 points (VI).

Let $\delta$ cut $c_{4}$ twice, then $\delta$ is a triple line; the residual curve is of order 3 , it cuts $\delta$ in 2 points (III).

Let $\delta$ cut $c_{4}$ three times; $\delta$ counts as a fourfold line and there is no other nodal curve on the surface (I).

Let $c_{4}$ be replaced by a double conic $c_{2}{ }^{2}$; the double curve consists of the conic and a twisted quartic which is cut by every generator twice (VIII).

Thus far the correspondence is between a line and a curve of order 4 ; it remains to consider the correspondence between a conic $c_{2}$ and a unicursal cubic $c_{3}$. The general case gives nothing new from the standpoint of the double curve.

* "Ueber die geradlinigen Flächen fünften Grades " Crelle, vol. 67.

$\dagger$ The Roman numerals refer to types given by Schwarz. 
Let $c_{3}$ be replaced by a triple line and $c_{2}$ by a double line; this accounts for a nodal curve of order 4 ; a unicursal quintic has a nodal line of order 6 , hence 2 double generators must exist $(\mathrm{V})$. This type can be defined by a $(2,3)$ correspondence between the points of two lines which are skew to each other, the correspondence to have one double element.

Let $c_{3}$ be replaced by a triple line ; the plane of $c_{2}$ will cut three generators from the surface, all of which pass through the trace $T$ of the triple line. Let one generator cut $c_{2}$ in $P_{1}$ and $P_{2}$; if $P_{1}$ corresponds to $T$ and $P_{2}$ corresponds to $T$, the generator joining $P_{1} P_{2}$ becomes double and the double curve consists of the triple line, the double generator, and a conic not in the plane of $c_{2}$ (IV).

Let $c_{2}$ be replaced by a double line; the plane of $c_{3}$ contains two generators, which may coincide as in the last case. The double curve consists of the double line, a double generator, and a twisted quartic which cuts the double generator in two points (VII).

Let $c_{2}$ be replaced by a double line; in general there is one point from which issue three pairs of generators; let this triple point be resolved, which can always be done by changing the parameters. The double curve is now a quintic with a double point (X).

Finally, let there be a $(2,2)$ correspondence between two conics which intersect in a double element. As each generator can cut each conic but once, but must cut the complete nodal curve three times, and as the nodal curve is of order 6 , it follows that the residual curve is a third conic section (IX).

From this method of generation the equations can be at once written down in the most general form.

Special forms of these types may exist : thus, in (I) $\delta$ may be (1) a fourfold directrix, (2) a triple directrix and a single generatur, (3) a double directrix and a double generator, (4) a single directrix and a threefold generator; all of these may be obtained in the same way by letting the directing line intersect the directing curve in the proper number of points. (II) may have (5) a line for simplest curve on the surface, or (6) a conic. In (III), the triple line may be (7) triple directrix, (8) a double directrix and single generator, or (9) a single directrix and double generator. Similarly in (IV), we have types (10), (11), (12). The last case presents a surface having a singularity not met with in scrolls of lower order. Let a $(1,2)$ cor. respondence exist between the points of a line and a conic 
which have one point in common. The line will be simple directrix and double generator. For example, let

$$
\begin{aligned}
& x=0, \quad y=0, \quad z=\frac{1}{\mu} \text { be the equations of the line, } \\
& x=\lambda^{2}, \quad y=\lambda, \quad z=0 \quad \text { be the equations of the conic; }
\end{aligned}
$$

and let $\lambda=\mu^{2}$. The equation of the scroll is

$$
y\left(x w-y^{2}\right)^{2}=x^{3} z^{2} .
$$

It is unicursal ; every generator cuts one other generator at a point on the conic and two others at the point in which it intersects the line. The triple line $x=0, y=0$ and the double conic $z=0, y^{2}=w z$ make a nodal curve of order 5 ; hence a double generator exists. But $x=0, y=0$ is a single directrix ; hence but one generator can pass through each point. The directrix line counts for a curve of order 4 as a component of the nodal sextic, though any plane through it will cut two generators from the surface. Any plane section not through a generator will cut the surface in a unicursal quintic curve having a tacnode and a simple branch through it at the trace of the directrix line; hence it can have but two other nodes, namely, at the points in which the cutting plane intersects the double conic. In (V) the directrices may be (13) distinct, or (14) coincident. In (VI) the line may be (15) double directrix, or (16) simple directrix and generator. In (VII) there are no subdivisions (17); nor in (VIII), (18); nor in (IX), (19). In (X) there are two possible types (20), (21).

\section{B. Quintics of Genus 1 .}

By joining corresponding points of two non-singular cubics which have one self-corresponding point, a quintic scroll of genus 1 is generated. The nodal curve is of order 5 (XI). There are no subdivisions of this form (22).

One cubic may be replaced by a triple line, leaving a conic for residual nodal curve (XII), (23). The double conic may degrade into a double generator and a double directrix line (XIII); the directrices may be (24) distinct or (25) coincident.

A double line can be brought intó $(1,1)$ correspondence with a non-singular cubic; the scroll thus defined has the double line and a twisted quartic for nodal curves (XIV) (26). 


\section{Quintics of Genus 2.}

The cubic may be replaced by a triple line; the nodal curve is completed by one double directrix and one triple directrix $(\mathrm{XV})$; the skew directric es may be (27) distinct or (28) coincident.

CORNeLl UNIVERSITY, January 25, 1902.

\section{SIMPLIFIED DEFINITION OF A GROUP.}

BY DR. E. V. HUNTINGTON.

(Read before the American Mathematical Society, February 22, 1902.)

UP to the present time no attempt seems to have been made to prove the independence of the postulates employed to define a group, and as a matter of fact the definition usually given contains several redundancies.* These redundancies are removed in the following note, the number of necessary portulates being reduced to three, and the independence of these three being established.

\section{Fundamental Concepts.}

A class of objects is determined when any condition is given such that every object in the universe must either satisfy or not satisfy the condition. Every object which satisfies the condition is said to belong to the class. (We shall agree to exclude the case of a class to which no element belongs.)

A class thus defined is usually called, in mathematical parlance, an assemblage (Menge, ensemble), every object which belongs to the class being called an element of the assemblage.

A rule of combination in an assemblage is any rule or agreement by which, when any two elements (whether the same or different) are given, in a definite order, some object (which may or may not itself belong to the assemblage ${ }^{\dagger}$ ) is uniquely determined.

If the first of the two given elements is denoted by $a$ and the second by $b$, then the object which they determine is denoted by $a \circ b$ (read: " $a$ with $b$ ").

\footnotetext{
* See for example H. Weber, Algebra, Vol. II. (1899), pp. 3-4.

+ The object determined by any two elements of the assemblage always will belong to the assemblage if postulates $1,2,3$ are satisfied, as we prove below in 10 .
} 